\title{
Qinghua Fang inhibits high-fat diet-induced non-alcoholic fatty liver disease by modulating gut microbiota
}

\author{
Yiqun Wang ${ }^{1 \#}$, Shengxia $\mathrm{Lv}^{2 \#}$, Tianbai Shen ${ }^{1}$, Minchao $\mathrm{Ye}^{1}$, Dehe Wang ${ }^{1}$, Pan Zhou ${ }^{1}$, Ying $\mathrm{Li}^{1}$, \\ Wei Zhang ${ }^{1}$
}

${ }^{1}$ Department of Hepatology, Longhua Hospital Affiliated to Shanghai University of Traditional Chinese Medicine, Shanghai, China; ${ }^{2}$ Department of Hepatology, Xi'an Hospital of Traditional Chinese Medicine, Xi'an, China

Contributions: (I) Conception and design: Y Li, W Zhang; (II) Administrative support: W Zhang; (III) Provision of study materials or patients: Y Wang, Y Li; (IV) Collection and assembly of data: Y Wang, S Lv, T Shen, M Ye, D Wang, P Zhou; (V) Data analysis and interpretation: Y Wang, S Lv; (VI) Manuscript writing: All authors; (VII) Final approval of manuscript: All authors.

\#These authors contributed equally to this work.

Correspondence to: Dr. Ying Li. Department of Hepatology, Longhua Hospital Affiliated to Shanghai University of Traditional Chinese Medicine, No. 725 South Wanping Road, Shanghai 200032, China. Email: 13817270542@163.com; Dr. Wei Zhang. Department of Hepatology, Longhua Hospital Affiliated to Shanghai University of Traditional Chinese Medicine, No. 725 South Wanping Road, Shanghai 200032, China. Email: 18918104444@189.cn.

Background Disturbance of the gut microbiota may play a critical role in the progression of nonalcoholic fatty liver disease (NAFLD) induced by high-fat diet (HFD). Changes in gut microbiota were analyzed in a rat HFD-induced NAFLD model following treatment with Qinghua Fang (QHF), a Chinese herbal formula currently used in the clinical practice of traditional Chinese medicine.

Methods: Sixty Wistar rats were randomly divided into 6 groups $(\mathrm{n}=10)$ : blank group [normal chow (NC) group], model group (HFD group), control group (BG group), Qinghua Fang high-dose group [QHF(H) group], QHF mid-dose group [QHF(M) group], QHF low-dose group [QHF(L) group]. The high, medium and low doses of QHF were used to intervene in the $\mathrm{H}, \mathrm{M}$, and $\mathrm{L}$ groups; the $\mathrm{BG}$ group was given berberine; the NC and HFD groups were given distilled water for 10 weeks. H\&E staining, determination of serum liver function and blood lipid levels, and changes in the structure of rat intestinal flora through $16 \mathrm{~S}$ rDNA denaturing gradient gel electrophoresis sequencing technology and ERIC-PCR fingerprinting were performed.

Results: The liver function and blood lipid levels of the rats in the HFD group were higher than those in the NC group; the alanine aminotransferase levels in the QHF-H group, QFH-M group and QHF-L group were lower than in the HFD group $(\mathrm{P}<0.05)$; the liver pathology of the QHF-M group and QHF-H group showed a small amount of fatty cell infiltration, but was significantly less than the hepatocyte inflammation and necrosis in the HFD group. The ERIC-PCR fingerprint and diversity analysis found that the composition of the intestinal flora of rats in the QHF-H group was significantly different from that of the NC and HFD groups. The flora of the QHF and NC groups was more diverse and richer than in the HFD group $(\mathrm{P}<0.05)$.

Conclusions: QHF alleviated the liver dysfunction and increased blood lipid levels of NAFLD rats induced by HFD. It also effectively reduced the degree of liver steatosis and adjusted the number and structure of intestinal flora. Treatment with QHF had a significant effect on NAFLD.

Keywords: Gut microbiota; nonalcoholic fatty liver disease (NAFLD); Chinese herbal formula; high-fat diet (HFD)

Submitted Jan 11, 2021. Accepted for publication Mar 20, 2021.

doi: 10.21037/apm-21-448

View this article at: http://dx.doi.org/10.21037/apm-21-448 


\section{Introduction}

Nonalcoholic fatty liver disease (NAFLD) has become one of the most common chronic liver diseases in the world, and is closely related to obesity, hyperlipidemia, diabetes mellitus and hypertension (1). NAFLD is defined as a spectrum of liver pathologies that are initiated by steatosis and may progress to nonalcoholic steatohepatitis (NASH), which includes necro-inflammation and fibrosis (2). NASH can progress to cirrhosis and eventually hepatocellular carcinoma (3). The global incidence of NAFLD in the general population ranges from $28.01 / 1,000$ to $52.34 / 1,000$ person-years (4,5). A meta-analysis indicated a global prevalence of NAFLD of approximately $25.24 \%$, with the highest prevalence in the Middle East, which reached $31.79 \%$, followed by South America at $30.45 \%$, and the lowest prevalence in Africa at $13.48 \%$ (5). About $25 \%$ of adults in the USA have NAFLD (6). The prevalence rate of NAFLD in Asia is about $25 \%$, and about $8-19 \%$ of the Asian people with a body mass index less than $25 \mathrm{~kg} / \mathrm{m}^{2}$ have NAFLD that is often referred to as "lean" or "nonobese" NAFLD (7). These data show the necessity of understanding this disease pathology on global health. In addition to genetic background, environmental factors, such as diet, exercise, shift work and alterations in commensal microbiota, should be considered significant in the development and progression of NAFLD $(6,8,9)$.

Gut microbiota is the unity of the interaction between the intestinal flora and its host. It is composed predominantly of four phyla: Firmicutes, Bacteroidetes, Actinobacteria, and Proteobacteria (10). The intestinal tract is home to $10^{13}-10^{14}$ micro-organisms, which exceeds the total number of body cells $\left(10^{13}\right)$ by a factor of $10(10)$. The gut microbiota has over 1,000 species, approximately $90 \%$ of the microbes belong to the Firmicutes and Bacteroidetes phyla. The ratio of Firmicutes/Bacteroidetes is considered to be an important factor affecting gut microbiota disorders, a significant increase in this ratio could also be regarded as a microbial marker signal of obesity and lipid deposition (11). The gut microbiota performs two main functions: energy metabolism and immune function. Energy metabolism includes the synthesis of all B vitamins (B1-B12) and vitamin $\mathrm{K}$ (12), decomposition of complex polysaccharides (e.g., starch, cellulose and pectin), synthesis of essential amino acids, production of short-chain fatty acids, and the provision of energy for the anti-inflammatory effects of colon bacteria (13). Furthermore, the gut microbiota is the first line of defense against pathogens and toxins. This function is modulated by direct interaction with the intestinal epithelial cells that constitute the mucosal immune system and other attachment sites, leading to the secretion of toxins to inhibit external bacterial invasion by intestinal competition at the mucosa.

In recent years, more and more evidence has shown that intestinal flora is an important factor in the occurrence and development of NAFLD (14). The intestinal flora can stimulate the absorption of glucose in the host intestine, increase the glucose and insulin content in the serum, thereby up-regulating the two basic transcription factors: carbohydrate response element binding protein (ChREBP) and sterol regulatory element binding protein (SREBP-1), accelerate the induction of liver fat synthesis and aggravate the development of NAFLD (15). The disorder of the intestinal barrier environment and the imbalance of the microbiota can participate in lipid regulation and the pathogenesis of NAFLD through pro-inflammatory response and bile acid metabolism, and the degree of flora disorder is positively correlated with the severity of fatty liver disease (14,16-18). The change of intestinal flora and the increase of intestinal permeability will increase the liver's exposure to intestinal-derived bacteria, which quickly transfer to the liver through the portal vein, induce the excessive activation of Kupffer cells, hepatic stellate cells, interleukins, tumor necrosis factor, other immune cells and pro-inflammatory factors in the liver (19-21). This leads to more severe liver damage, inflammation and fibrosis, promotes changes in the related gut-liver axis and accelerates the development of NAFLD $(21,22)$.

The maintenance of balance in the gut microbiota is important for health and treatment of diseases, and modulating microbiota has become a therapeutic approach. Traditional Chinese medicine (TCM) has been used for thousands of years in China, and most TCM prescriptions are decoctions. Recent studies have shown that the effective components of many herbs can only be absorbed through the intestines and they play their biological roles according to the host intestinal microflora (23). Furthermore, some current data show that TCM herbs may modulate the gut microbiota to some extent (24-26). Mei et al. (27) have found that the combined intervention of anthraquinone of Cassia obtusifolia and cholesterol-lowering probiotics can improve the intestinal mucosal barrier function, reduce triglycerides and cholesterol and inhibit free fatty acids, thereby improving the NAFLD process; Peng et al. (28) have found that the geniposide-chlorogenic acid composition of the Chinese medicine Qushi Huayu Decoction can improve liver fat by repairing the intestinal 
mucosal barrier and adjusting the disturbance of intestinal flora, which has a good therapeutic effect on NAFLD. Qinghua Fang (QHF), a Chinese herbal formula, is based on Buzhongyiqi Tang, and has shown significant effects in the clinical treatment of NAFLD. In the preliminary clinical randomized controlled experimental study, QHF can effectively reduce the BMI index, liver CAP value, serum alanine aminotransferase (ALT) and triglyceride (TG) levels in patients with NAFLD and have a good curative effect on NAFLD (29). The main ingredients of QHF, such as Rbizoma atractylodis macrocephalae and Wolfiporia cocos, have been included in the "Chinese Medicinal Diet Dictionary" and been used as common medicinal diets for the patient's physical fitness and supplement treatment (30). In our preliminary experiment (31), there was little fatty infiltration in the liver cells of the QHF treatment group, and the composition and distribution of the bands of the ERIC-PCR fingerprint in the treatment group were more numerous and wider than in the model group. The similarity of intestinal flora between the blank group and treatment group was greater than that between the blank and model groups and between the model and treatment groups. Previous studies have suggested that the main components of QHF can alter the intestinal microbiota and improve lipid metabolism; for instance Gynostemma pentaphyllumcan be increased beyond the relative abundance of Clostridium cocleatum (25), and Rbizoma Atractylodis macrocephalae can modulate the gut microbial distribution (26). $\mathrm{Xu}$ et al. (32) have found that Danshensu Bingpian Zhi, which is derived from Salvia miltiorrhiza, is a putative PPAR $\gamma$ agonist that reverses gut microbiota dysbiosis and improves high-fat diet (HFD)-induced metabolic syndrome. Tung et al. (33) have discovered that citrus peel extracts can alter the intestinal microbiota by adding Prevotella sp. reducing rc4-4 bacteria and decreasing lipid accumulation. Yang et al. (34) have found that lotus leaf has a protective effect for NAFLD by improving insulin resistance and restraining the inflammatory reaction. Thus we inferred that QHF can effectively alleviate injury of liver cells and improve steatosis in NALFD, and also can modulate the intestinal flora. However, there has not been a credible and systematic explanation of its mechanism of action. The ability of QHF to modulate the host's gut ecosystem and alleviate the symptoms of NAFLD and the potential of the gut as a target for QHF remain to be clarified.

Early studies have shown changes in the intestinal microbiota in metabolic syndrome and fatty liver hereditary obese mice, and those studies associated abnormal microbial populations with inflammatory signals that promote insulin resistance, liver fatty degeneration, and NAFLD (35-37). Follow-up work indicated that the gut-liver axis has important bidirectional effects on health (38-41). At the same time, the imbalance of intestinal flora is closely related to the disorder of blood lipid metabolism in the process of transforming NAFLD to primary liver cancer (42). Previous studies have shown that taking dietary supplements that restore intestinal function and microbial community could improve liver lipids deposition and systemic inflammation, and be conducive to the normal metabolism of cholesterol $(42,43)$. It is still an uncertain, but exciting possibility, that insights gained from animal studies can inform human efforts to prevent or treat NASH (6). The rat model of NAFLD induced by HFD is recognized as valid and is widely used in experimental studies (44). In addition to changes in liver histology, the obesity and slow action of NAFLD rats are similar to the clinical symptoms of NAFLD patients. Therefore, we selected the NAFLD rat model induced by HFD for our study. We also applied advanced techniques of polymerase chain reactiondenaturing gradient gel electrophoresis (PCR-DGGE) and bar-coded pyrosequencing to clearly observe changes in the composition of the intestinal microflora after QHF treatment. The purpose of this study was to determine whether QHF was effective in the treatment of NAFLD, and the effects on intestinal microbiota. We present our findings in accordance with the ARRIVE reporting checklist (available at http://dx.doi.org/10.21037/apm21-448).

\section{Methods}

\section{QHF preparation}

QHF used in the present study was prepared from the following herbs: Sedum sarmentosum $15 \mathrm{~g}$, Radix salvia $9 \mathrm{~g}$, Rhizoma atractylodis macrocephalae $12 \mathrm{~g}$, Tangerine peel $9 \mathrm{~g}$, Lotus leaf $15 \mathrm{~g}$, Gynostemma pentaphyllum $9 \mathrm{~g}$, Wolfiporia cocos $9 \mathrm{~g}$, Eupatorium japonicum $15 \mathrm{~g}$, Rbizoma alismatis $15 \mathrm{~g}$, and Stigma maydis $9 \mathrm{~g}$. The herbs were obtained from the Pharmacy Department of Longhua Hospital Affiliated to Shanghai University of Traditional Chinese Medicine, and all herbs met the standards specified in the Chinese Pharmacopoeia (1995 edition). According to the standard of Chinese pharmacological research methodology, the herbs were mixed and added to $600 \mathrm{~mL}$ distilled water and decocted twice; the two decocted liquids were combined and filtered through four layers of gauze, incubated in a 
water bath at $95{ }^{\circ} \mathrm{C}$, concentrated to a volume of $555.5 \mathrm{~mL}$, cooled and then stored at $4{ }^{\circ} \mathrm{C}$ until use.

\section{Ethics statement}

All procedures for animal use and care in the present study were reviewed and approved by the Animal Ethics Committee of the Medical Center of Fudan University (Shanghai, China; Approval No. 20171717A744). The care and use of animals followed the Guidelines for Animal Experiments of the Medical Center of Fudan University, and all efforts were made to minimize the number of animals and their suffering (45).

\section{Animals}

Sixty male Wistar rats, weighing $150-170 \mathrm{~g}$, were purchased from the Shanghai Sippr-BK Laboratory Animal Co. Ltd. (Shanghai, China) and housed in a specific pathogen-free barrier system in the Laboratory Animal Center of the Medical Center of Fudan University, under a standard $12 \mathrm{~h}$ light $/ 12 \mathrm{~h}$ dark cycle. The temperature and relative humidity of the animal room were set at $23 \pm 2{ }^{\circ} \mathrm{C}$ and $62 \% \pm 5 \%$, respectively. All the rats were kept in plastic cages with clean sawdust pads. Four rats were placed in each cage, and the bedding was replaced twice weekly. Water was provided by water bottles with high-pressure sterilization and they were refilled 2-3 times weekly to ensure continued supply of drinking water. After acclimatization for 1 week, the rats were randomly divided into six groups ( $\mathrm{n}=10 /$ group): five of these groups were fed a HFD (88\% regular feed, $10 \%$ lard, and $2 \%$ cholesterol), and a control group was fed normal chow (NC). QHF was administered to the rats at $1 \mathrm{~mL} / 100 \mathrm{~g}$ (equivalent to the human dose). Three of the HFD groups were given QHF by oral gavage at three dose levels: high $(\mathrm{H})$, middle $(\mathrm{M})$ and low $(\mathrm{L})$ levels $(0.80,0.40$, and $0.20 \mathrm{~g} / 100 \mathrm{~g}$ body weight, respectively). One HFD group was given berberine (daily dose of $10 \mathrm{mg} / 100 \mathrm{~g}$ body weight) obtained from Northeast China Pharmaceutical Group Shenyang First Pharmaceutical Co. Ltd. (batch no. 201500505). The fifth HFD group and the NC group were given control vehicle (distilled water at the same volume. Food intake was controlled to the same level for all the HFD rats. Body weight was measured every 7 days. All the rats were starved for $8 \mathrm{~h}$ before being killed humanely by intraperitoneal injection of $2 \%$ pentobarbital sodium $(30 \mathrm{mg} / \mathrm{kg}$ ) and then weighed. The abdominal cavity was opened for drawing blood samples and removal of the liver. Part of the liver was rapidly placed in liquid nitrogen, and stored for further analysis. A piece of liver $(\approx 2 \times 1 \mathrm{~cm})$ was cut from the middle section of the liver. Paraffin sections were prepared after $10 \%$ formaldehyde fixation for hematoxylin-eosin (H\&E) staining. Abdominal aorta blood samples were used for analyses of liver function, blood lipids and cytokines. Alanine aminotransferase (ALT), aspartate aminotransferase (AST), gamma glutamyl transpeptidase $(\gamma-G T)$, triglycerides (TG), total cholesterol (TC), highdensity lipoprotein cholesterol (HDL), low-density lipoprotein cholesterol (LDL) and lipopolysaccharide (LPS) were assayed using kits obtained from the Chinese Medicine (Group) Shanghai Chemical Reagents Company. Serum levels of tumor necrosis factor-alpha (TNF- $\alpha$ ), interleukin (IL)-6, IL-8, IL-17, and IL-10 and transforming growth factor- $\beta$ (TGF- $\beta$ )were determined by enzyme-linked immunosorbent assays, and the kit was purchased from Shanghai Nanxi Biotechnology Co., Ltd. Before death, fecal samples were collected from all the animals and stored at $-70{ }^{\circ} \mathrm{C}$ until analysis.

\section{Amplification of bacterial $16 S \mathrm{rDNA}$ fragments by PCR}

The stool samples were labeled as follows: A1-A9 (HFD control), B1-B9 [QHF (M)], C1-C10 [QHF (L)], H1H10 (berberine), K1-K10 (NC) and M1-M10 (HFD). ERIC-PCR fingerprint detection and DGGE analysis were performed by Shanghai Rui Jin Biotechnology Co., Ltd. Universal bacterial universal primers GC-338F (5'-CGCC CGGGGCGCGCCCCGGGGCGGGGCGGGGGCG CGGGGGGCCTACGGGAGGCAGCAG-3') and 518R (5'-ATTACCGCGGCTGCTGG -3') were used to amplify $16 \mathrm{~S}$ rRNA hypervariable region sequences.

The PCR amplification system $(50 \mu \mathrm{L})$ comprised the following: $5 \mu \mathrm{L}$ of $10 \times$ PCR buffer; $3.2 \mu \mathrm{L}$ of DNTPs $(2.5 \mathrm{mmol} / \mathrm{L}) ; 0.4 \mu \mathrm{L}$ of rTaq $(5 \mathrm{U} / \mu \mathrm{L}) ; 1 \mu \mathrm{L}$ of $\mathrm{GC}-338 \mathrm{~F}$ $(20 \mathrm{mmol} / \mathrm{L}) ; 1 \mu \mathrm{L}$ of $518 \mathrm{R}(20 \mathrm{mmol} / \mathrm{L}) ; 50 \mathrm{ng}$ of template DNA; adding $\mathrm{ddH}_{2} \mathrm{O}$ to $50 \mu \mathrm{L}$ ). The PCR amplification was performed using the following thermocycling program: predenatured for $5 \mathrm{~min}$ at $94{ }^{\circ} \mathrm{C}$ and denatured for $1 \mathrm{~min}$ at $94{ }^{\circ} \mathrm{C}$, followed by 30 cycles of annealing $45 \mathrm{~s}$ at $55^{\circ} \mathrm{C}$, and extension for $1 \mathrm{~min}$ at $72{ }^{\circ} \mathrm{C}$, with a final extension for $10 \mathrm{~min}$ at $72{ }^{\circ} \mathrm{C}$. The PCR products $(10 \mu \mathrm{L})$ were analyzed by DGGE using a polyacrylamide gel with a gradient of $35-55 \%$ and a concentration of $8 \%$ (chemical denaturant: $100 \%$ urea, $7 \mathrm{~mol} / \mathrm{L}$ and $40 \%(\mathrm{v} / \mathrm{v})$ acrylamide for $5 \mathrm{~h}$ $\left(150 \mathrm{~V}, 60{ }^{\circ} \mathrm{C}\right)$ in $1 \times$ TAE buffer solution. After silver staining, a sterilized scalpel was used to recover the 
DGGE bands and DNA was isolated using a PolyGel DNA Extraction Kit (Omega). PCR amplification was then carried out using $2 \mu \mathrm{L}$ of the product as a template, $338 \mathrm{~F} / 518 \mathrm{R}$ as the primers and the following thermocycling program: predenatured for $4 \mathrm{~min}$ at $94{ }^{\circ} \mathrm{C}$ and denatured for $30 \mathrm{~s}$ at $94^{\circ} \mathrm{C}$, followed by 30 cycles of refolding for $30 \mathrm{~s}$ at $55^{\circ} \mathrm{C}$ and $30 \mathrm{~s}$ at $72{ }^{\circ} \mathrm{C}$, with a final extension for $10 \mathrm{~min}$ at $72{ }^{\circ} \mathrm{C}$. The newly amplified fragments of DNA were recovered, purified and then ligated into the Pmd18T vector for transformation into DH5 a competent cells. The positive clones were screened and sequenced. The sequencing results were compared with the sequence in the GenBank, and the type of bacteria represented by the strip was obtained. Three clones were selected for sequence determination in each recovery strip.

\section{Diversity index analysis}

The number and density of bands in the DGGE profiles for each sample were analyzed digitally using Quantity One, and the Shannon diversity index $\left(H^{\prime}\right)$ and species richness diversity index $\left(D_{M g}\right)$ were used to compare the variety of different samples according to the following algorithm (46):

$$
\begin{aligned}
& H^{\prime}=-\sum_{i=1}^{S} p i \ln p i=-\sum_{i=1}^{S}(N i / N) \ln (N i / N) \\
& D_{M g}=\frac{S-1}{\log \sum_{i=1}^{S} n_{i}}
\end{aligned}
$$

where $p i$ is the ratio of single-band intensity samples accounted for in the sample of all bands in total intensity, $N$ is the all-bands abundance of a single lane on the DGGE profiles, $N i$ is the abundance of the $i$-th band, and $S$ is the sum of all bands in a sample.

\section{Statistical analyses of the serum index, Shannon diversity index and richness diversity index}

Data analysis was performed using IBM SPSS statistics software (version 21, IBM Corporation, Shanghai, China). All the data were tested for normal distribution prior to analysis. ANOVA was used for comparisons of three or more samples that were independently considering two factors. Post-hoc tests (Least Significant Difference Tests, LSD tests) were used for comparisons between two independent groups. Variables are reported as mean and standard deviation (mean $\pm \mathrm{SD}$ ). Differences were considered statistically significant at $\mathrm{P}<0.05$.

\section{Similarity clustering analysis of the rats in each group and correlation coefficients}

Similarity clustering analysis of the rats in each group was performed using the POPGENE program (47). Because the data were not normally distributed, the nonparametric Spearman's rank correlation was used to determine correlation coefficients between the gut microbiota and the inflammatory factors of the HFD group, and the correlation coefficients between the gut microbiota and the serum lipids of the HFD group (48).

\section{Results}

\section{Reduction in body weight gain and alleviation of NAFLD in rats treated with $Q H F$ and berberine}

The rats in each group had normal mental state, good appetite, and shiny fur. Unfortunately, two rats died (one each in the $\mathrm{QHF}(\mathrm{H})$ and $\mathrm{QHF}(\mathrm{M})$ groups) at 10 weeks after oral gavage. At the end of the 10-week intervention period, rats in the NC group weighed significantly less than those in the HFD control group $(\mathrm{P}<0.01 ; 350.68 \pm 30.90$ vs. $393.36 \pm 32.34 \mathrm{~g}$, respectively; Figure $1 A$ ). The rats in the $\mathrm{QHF}(\mathrm{H}), \mathrm{QHF}(\mathrm{M})$ and berberine (BG) groups also weighed significantly less than those in the HFD control group $(\mathrm{P}<0.01,372.44 \pm 48.66$ vs. $393.36 \pm 32.34 \mathrm{~g}, \mathrm{QHF}(\mathrm{H})$ vs. HFD; $\mathrm{P}<0.01,378.62 \pm 39.35$ vs. $393.36 \pm 32.34 \mathrm{~g}, \mathrm{QHF}(\mathrm{M})$ vs. HFD; $\mathrm{P}<0.01,386.77 \pm 36.88$ vs. $393.36 \pm 32.34 \mathrm{~g}$, BG vs. HFD; Figure 1A).

Histological analysis (Figure $1 B$ ) showed that the liver tissue of rats in $\mathrm{NC}$ group was clear and complete, with normal hepatic lobule structure, neat hepatic cord arrangement, and polygonal-shaped hepatocytes. The nucleus was large and round, located in the center of the polygon, and the cytoplasm was uniform. There was no obvious hepatocyte steatosis and the portal area was not expanded. No fibroplasia or inflammatory cell infiltration was observed. In contrast, the liver tissue structure of the HFD and the QHF(L) groups was not clear. Although the structure of the hepatic lobules remained, the hepatic cord arrangement was disordered, and more than $75 \%$ of hepatocytes showed diffuse steatosis. The hepatocytes were obviously enlarged and the cytoplasm was loose. A large number of lipid droplets were found in the cytoplasm, and the nucleus was tilted to one side. No cirrhosis was found and occasional infiltrating inflammatory cells were observed. The liver structure in the berberine group was not clear, although the hepatic lobule structure remained. 


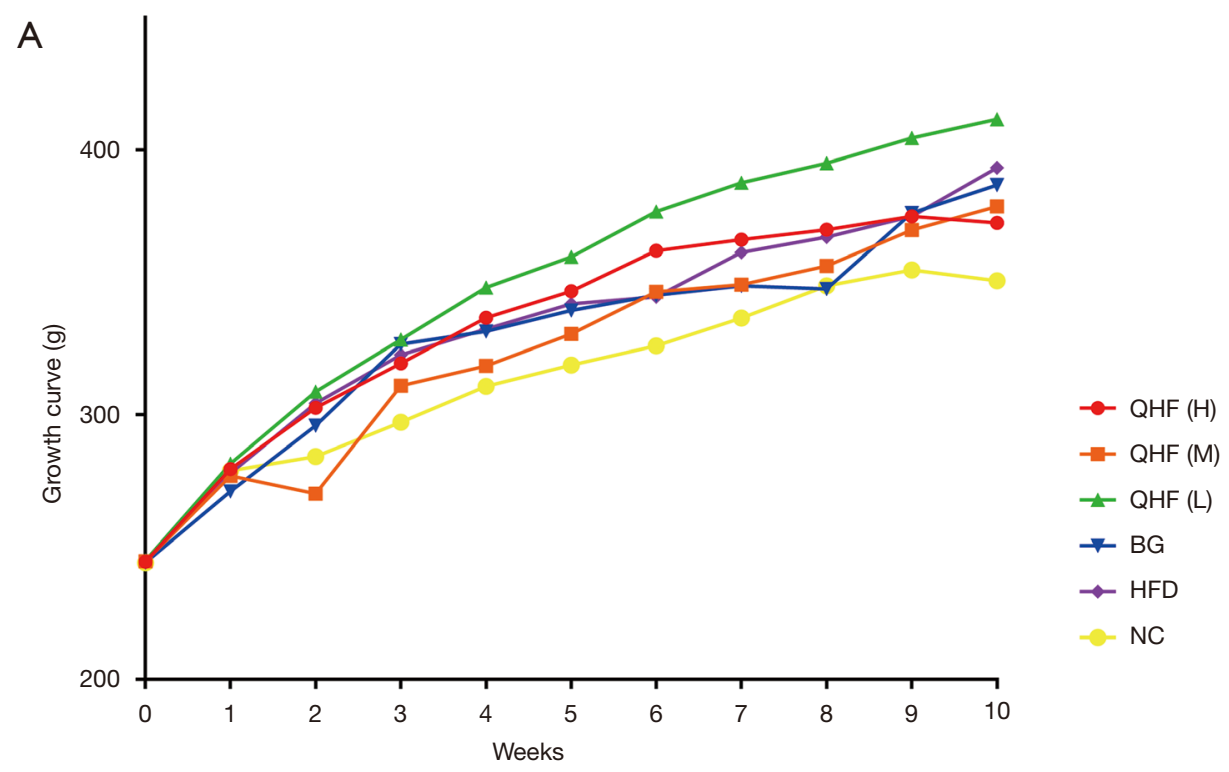

B
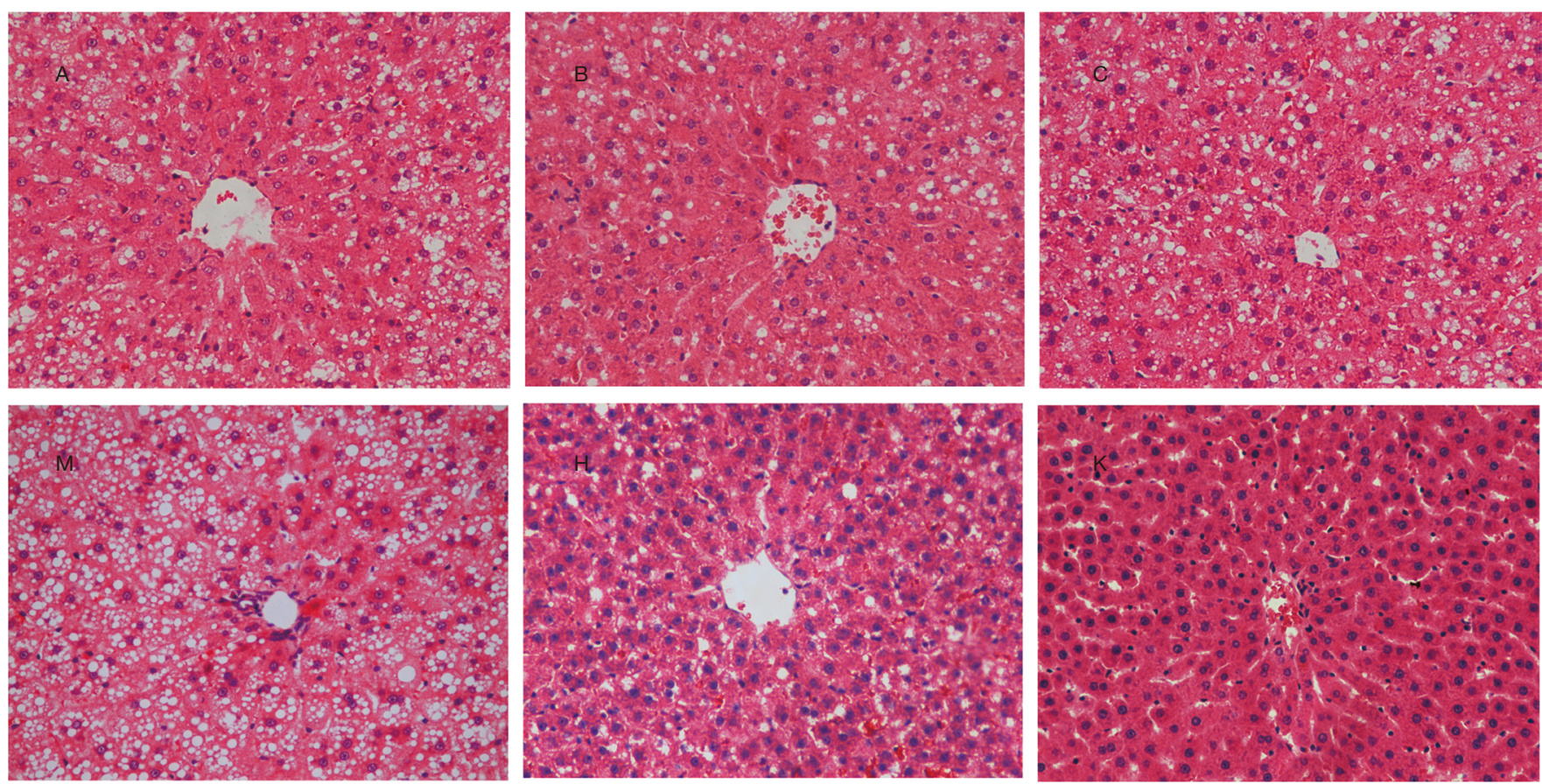

Figure 1 Alleviation of nonalcoholic fatty liver disease by treatment with Qinghua Fang (QHF) and berberine. (A) Body weight as measured weekly. (B) Hematoxylin-eosin (H\&E) staining of hepatocytes (magnification, ×200). A: QHF(H), QHF high-dose treatment group; B: QHF(M), QHF mid-dose treatment group; C: QHF(L), QHF low-dose treatment group; M: HFD, high-fat diet group; H: BG, berberine treatment group; K: NC, normal chow-fed group. 
A
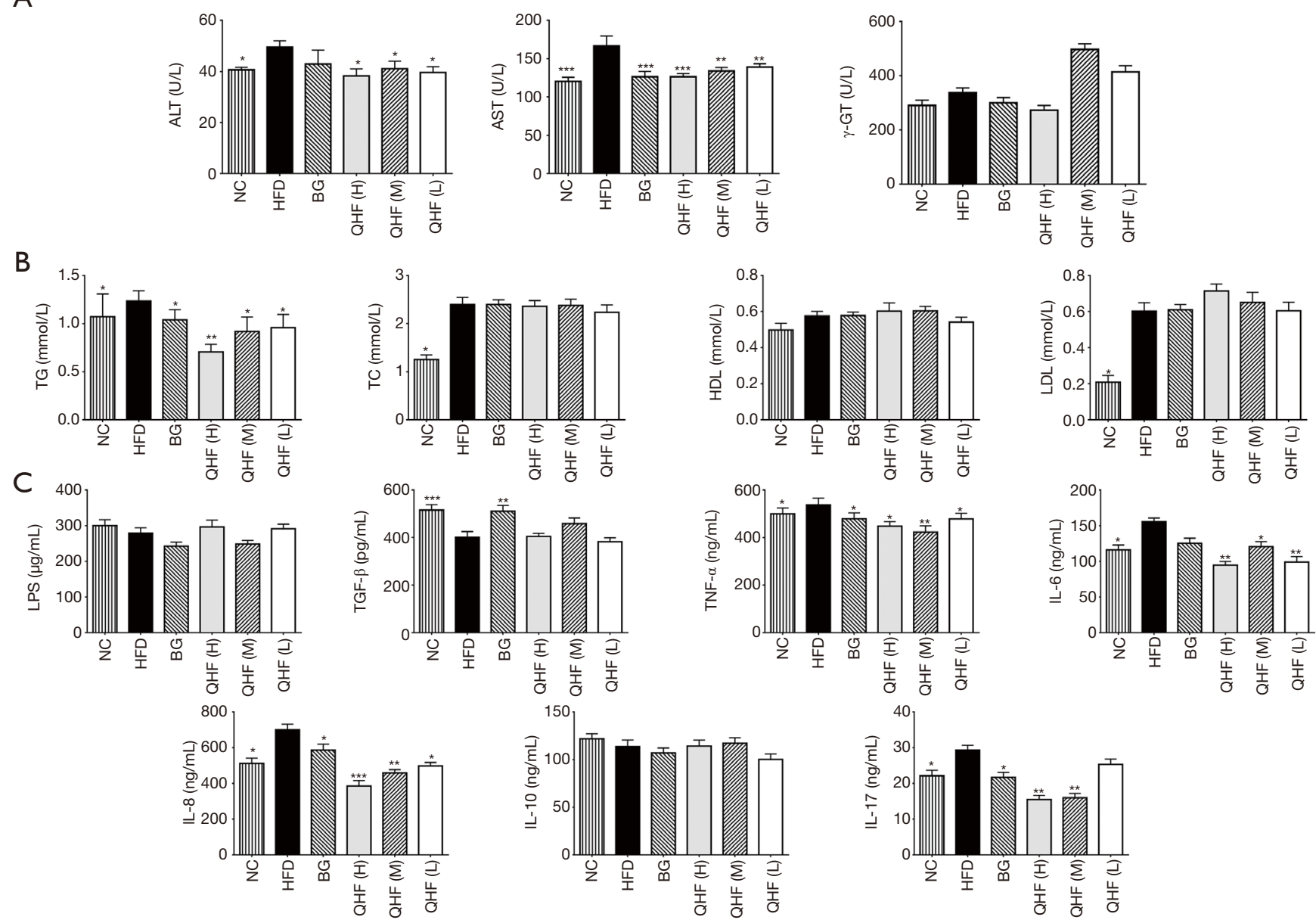

Figure 2 Serum indices in all groups at the end of 10 weeks: (A) liver function, (B) blood lipids and (C) inflammatory factors. Data are mean \pm standard deviation. ${ }^{*} \mathrm{P}<0.05,{ }^{* *} \mathrm{P}<0.01,{ }^{* *} \mathrm{P}<0.001$. ALT, alanine aminotransferase; AST, aspartate aminotransferase; $\gamma$-GT, gamma glutamyl transpeptidase; TG, triglycerides; TC, total cholesterol; HDL, high-density lipoprotein cholesterol; LDL, low-density lipoprotein cholesterol; LPS, lipopolysaccharide; TNF- $\alpha$ : tumor necrosis factor; TGF- $\beta$ : transforming growth factor- $\beta$; IL, interleukin; NC, normal chow; HFD, high-fat diet; QHF, Qinghua Fang; QHF(H), QHF high-dose treatment group; QHF(M), QHF mid-dose treatment group QH (L), QHF low-dose treatment group; BG, berberine treatment group.

Approximately $50-70 \%$ of hepatocytes showed diffuse steatosis, although no inflammatory cell infiltration was observed. The liver tissue structure of the $\mathrm{QHF}(\mathrm{M})$ and $\mathrm{QHF}(\mathrm{H})$ groups were still clear, and the hepatic lobule structure was intact. Approximately $30-50 \%$ of hepatocytes showed diffuse steatosis, although no inflammatory cell infiltration was observed.

\section{Determination of serum indexes}

The ALT and AST levels in the HFD group were significantly higher than in the $\mathrm{NC}$ group $(\mathrm{P}<0.05)$
(Figure 2A). There were no significant differences in the $\gamma$-GT indexes among the HFD, $\operatorname{QHF}(\mathrm{H}), \operatorname{QHF}(\mathrm{M})$ and $\mathrm{QHF}(\mathrm{L})$ groups $(\mathrm{P}>0.05)$.

The TG, TC and LDL levels were higher in the HFD group than those in the $\mathrm{NC}$ group $(\mathrm{P}<0.05)$ (see Figure $2 B$ ). There were no significant differences in the TC, HDL and LDL indexes between the HFD, $\mathrm{QHF}(\mathrm{H}), \mathrm{QHF}(\mathrm{M})$ and $\mathrm{QHF}(\mathrm{L})$ groups $(\mathrm{P}>0.05)$.

The levels of serum TNF- $\alpha$, IL-6, IL-8 and IL-17 in the HFD group were higher than in $\mathrm{NC}$ group $(\mathrm{P}<0.05)$ (Figure $2 C$ ). The IL-17 indices in the $\mathrm{QHF}(\mathrm{H})$ and $\mathrm{QHF}(\mathrm{M})$ groups were significantly lower than that in the 
A

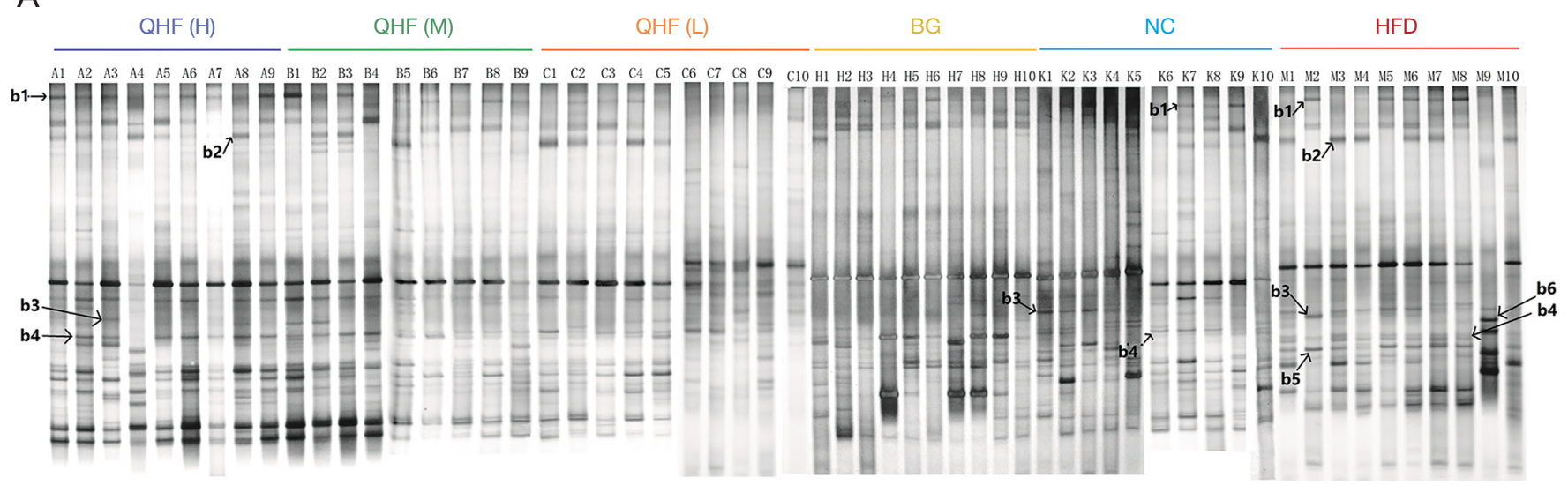

B

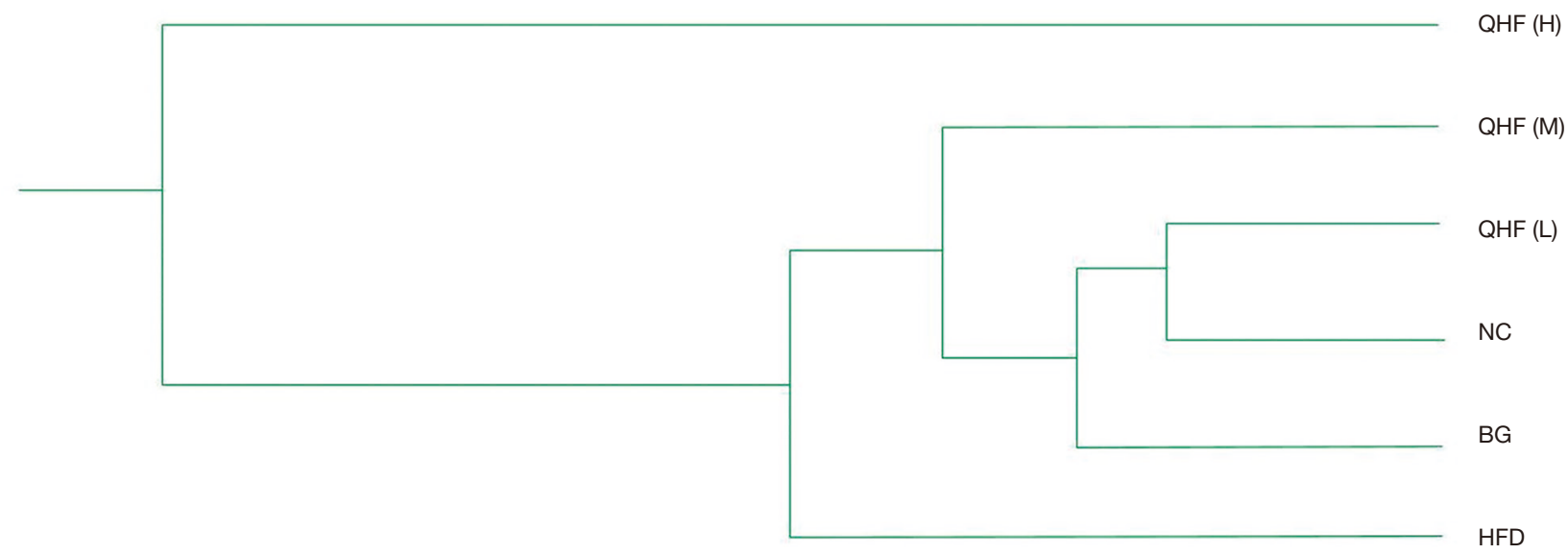

Figure 3 Comparison of gut microbiota by denaturing gradient gel electrophoresis (DGGE) fingerprinting in each rat group. (A) Structural changes in gut microbiota revealed by bar-coded pyrosequencing from fecal bacterial communities of the rats in each group. A1-A9 (QHF(H), QHF high-dose treatment group), B1-B9 (QHF(M), QHF mid-dose treatment group), C1-C10 (QHF(L), QHF low-dose treatment group), H1-H10 (BG, berberine treatment group), K1-K10 (NC, normal chow-fed group) and M1-M10 (HFD, high-fat diet group). The arrow means the band concerned by the research. (B) Similarity clustering analysis of the rats in each group.

HFD group $(\mathrm{P}<0.05)$. The TNF- $\alpha$, IL-6 and IL-8 indexes in the $\mathrm{QHF}(\mathrm{H}), \mathrm{QHF}(\mathrm{M})$ and $\mathrm{QHF}(\mathrm{L})$ groups were significantly lower than that in the HFD group $(\mathrm{P}<0.05)$.

\section{Comparison of gut microbiota profiles}

The intensities of bands 3 (b3) and b4 for the HFD-fed rats were significantly reduced, compared with the NC group. Sequence analysis showed that these bands were related to Flintibacter butyricus and Desulfotomaculum thermoacetoxidans, respectively. Our DGGE profiles demonstrated unique band patterns for the HFD group, which could be used to differentiate them from the other groups. The b5 and b6 bands were found only in the HFD-fed rats, and were related to Clostridium glycyrrbizinilyticum and Gemmiger formicilis, respectively (Figure $3 A$ ). Similarly, the DGGE banding patterns and cluster analysis indicated a significant difference in the gut microbiota composition between the QHF groups and the HFD group, as well as between the NC and HFD groups (Figure $3 A, B$ ). Interestingly, cluster analysis revealed markedly similar gut microbiota compositions in the treatment and NC groups (Figure 3B), 


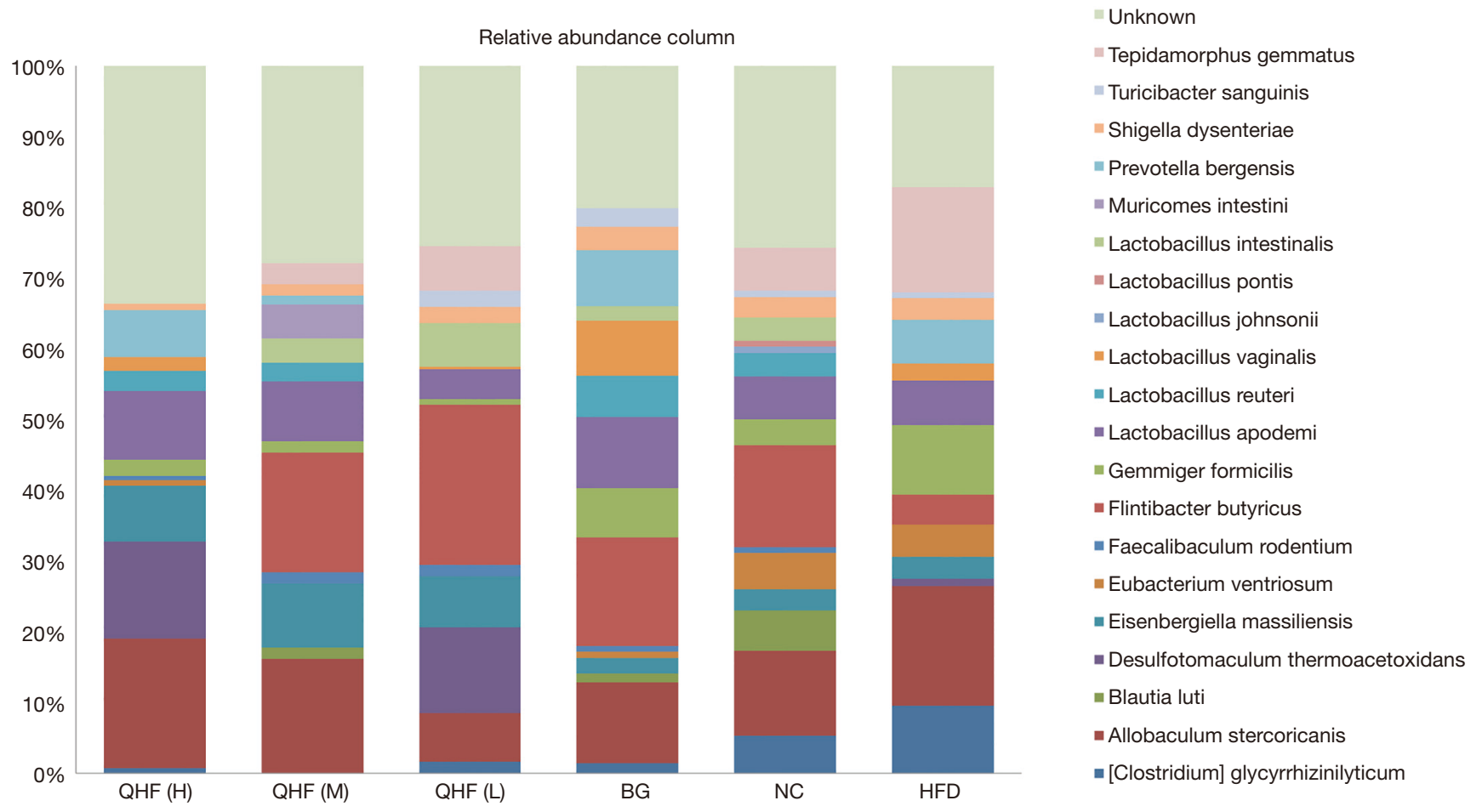

Figure 4 Abundance distribution of the 20 phylotypes identified as key classification variables.

while the gut microbiota of each QHF treatment group was significantly dissimilar to that of the HFD group. In our study, Flintibacter butyricus, Allobaculum stercoricanis and Lactobacillus apodemi were the dominant strains of gut microbiota, with very large proportions in each group, with the exception of Flintibacter butyricus in the $\mathrm{QHF}(\mathrm{H})$ and HFD groups (Figure 4). The Shannon diversity index and species richness analyses (Figure $5 A, B)$ showed that the gut microbiota of the QHF and NC groups was significantly more diverse and richer than that of the HFD group $(\mathrm{P}<0.05)$. Other intriguing questions we asked regarded the correlation between the gut microbiota and serum lipids, and between the gut microbiota and serum inflammatory factors in the HFD group. Spearman's rank correlation analysis was conducted to evaluate potential associations of the gut microbiota with serum lipids and serum inflammatory factors (Figure 6A,B). IL-17 and TG exhibited significant positive $(\mathrm{P}<0.05)$ correlations with Clostridium glycyrrbizinilyticum, whereas IL-8 and TG exhibited significant negative $(\mathrm{P}<0.05)$ correlations with Eisenbergiella massiliensis. Furthermore, a significant positive correlation $(\mathrm{P}<0.05)$ was observed between HDL and Lactobacillus apodemi, and a significant negative correlation $(\mathrm{P}<0.05)$ was observed between TNF- $\alpha$ and L. apodemi.
LDL and TC also exhibited significant positive correlations $(\mathrm{P}<0.05)$ with Lactobacillus vaginalis, and a significant negative $(\mathrm{P}<0.05)$ correlation was observed between TNF- $\alpha$ and $L$. vaginalis. Additionally, TGF- $\beta$ exhibited a significant positive correlation $(\mathrm{P}<0.05)$ with Allobaculum stercoricanis and LPS exhibited a significant positive correlation $(\mathrm{P}<0.05)$ with Eubacterium ventriosum.

\section{Discussion}

In the present study, the rats fed with HFD showed body weight gain and visceral obesity. The principal histopathological finding was hepatic steatosis, which was consistent with observations from other studies (49). Intragastric administration of QHF to HFD-fed rats might counteract increased liver weight by reducing steatosis, an effect that is derived from a reduction in plasma dyslipidemia. These results provide evidence for a protective effect of QHF against the increase in metabolic biomarkers observed in experimentally induced NAFLD. Previous studies have indicated that QHF can effectively adjust the gut microbiota, which accords with our results. For instance, Chen et al. (25) found that Gynostemma pentaphyllum increased the relative abundance of Clostridium 
A

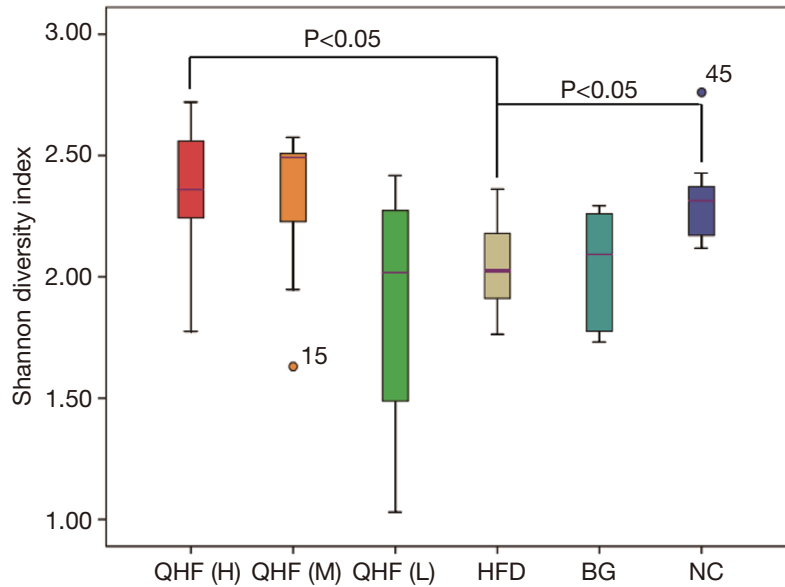

B

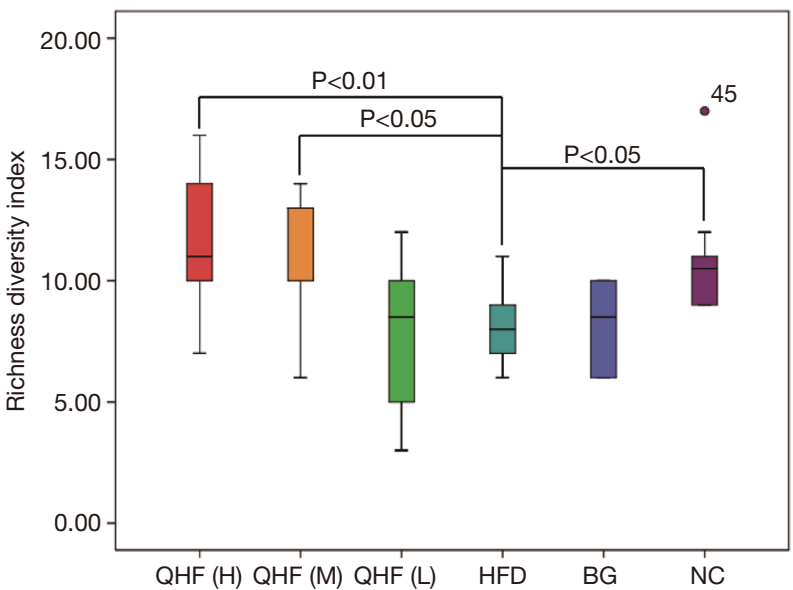

Figure 5 Box-plot of rat intestinal flora diversity. (A) Shannon diversity index of each rat group at the species level. (B) Richness diversity index of each rat group at the species level.

cocleatum and Bacteroides acidifaciens, which is beneficial to the host. Furthermore, Wang et al. (26) observed that Rbizoma atractylodis macrocephalae exhibits marked antiinflammatory, anti-adipogenic, and anti-obesity activities, and can modulate the gut microbial distribution. A number of previous studies have suggested that gut microbial metabolic interactions play a significant role in obesity, which is associated with induction of low-grade chronic systemic inflammation (50). Our results showed clearly the elevated serum levels of TNF- $\alpha$, IL-6, IL-8, and IL-17 in rats fed with HFD for 10 weeks, compared with those fed with a normal diet for the same period. Earlier studies have demonstrated that the pathogenesis of NAFLD is associated with increased intestinal endotoxemia and that LPS can lead directly to obesity and other complications through the interaction of the intestinal LPS-binding protein and the surface membrane CD14 receptor $(51,52)$. This leads to activation of Kupffer cells and nuclear factor (NF- $\mathrm{BB})$, followed by the release of pro-inflammatory factors, such as TNF- $\alpha$, IL-6, and IL-8, and the development of related inflammatory diseases (53).

The anti-inflammatory activities of QHF and berberine were further confirmed in the present study, resulting in significant decrease in the serum levels of TNF- $\alpha$, IL-6, IL-8, and IL-17 in the rats of the HFD-induced NAFLD group, while serum TGF- $\beta$ levels were increased. However, in the present study, $\mathrm{QHF}(\mathrm{H})$ exhibited significantly higher inhibition of the serum levels of TNF- $\alpha$, IL-6, IL-8, and IL-17, compared with the QHF(L) group. Based on these findings, it is conceivable that $\mathrm{QHF}(\mathrm{H})$ may be more effective than $\mathrm{QHF}(\mathrm{L})$ in the attenuation of systemic inflammation. Furthermore, our results showed that feeding rats a HFD for 10 weeks induced obvious obesity and NAFLD, as indicated by a significant increase in body weight and histopathological changes in adipose in the H\&E-stained hepatocytes, accompanied by increased serum levels of TC, TG, LDL, AST, and AST compared with the NC group. Furthermore, Clostridium glycyrrbizinilyticum was found only in the HFD-fed group, and the IL-17 and TG serum levels exhibited a significant positive correlation $(\mathrm{P}<0.05)$ with C. Glycyrrbizinilyticum, which is a member of the glycyrrhizin-hydrolyzing bacterium isolated from human feces and could be categorized as a beneficial Clostridium strain because of its multiple effects on the body study (54). However, the results of our experiment show that the presence of $C$. glycyrrbizinilyticum might be related to the increase in the serum lipid level and the promotion of an inflammatory response. Furthermore, the serum levels of ALT, AST and TG, TC and LDL were significantly decreased in the $\mathrm{QHF}(\mathrm{H}), \mathrm{QHF}(\mathrm{M})$, $\mathrm{QHF}(\mathrm{L})$ and berberine groups compared with in the HFD group. Based on these results, QHF might be effective in combating obesity and NAFLD by promoting lipid metabolism and inhibiting inflammatory responses.

Lye et al. (55) reported that hypercholesterolemic mice fed Lactobacillus fermentum FTDC 8312 showed a decline in serum TC levels, followed by a decrease in serum LDL levels and an increase in serum HDL levels. In addition, their study demonstrated that the cholesterol-lowering effect of L. fermentum FTDC 8312 may be by virtue of 
A

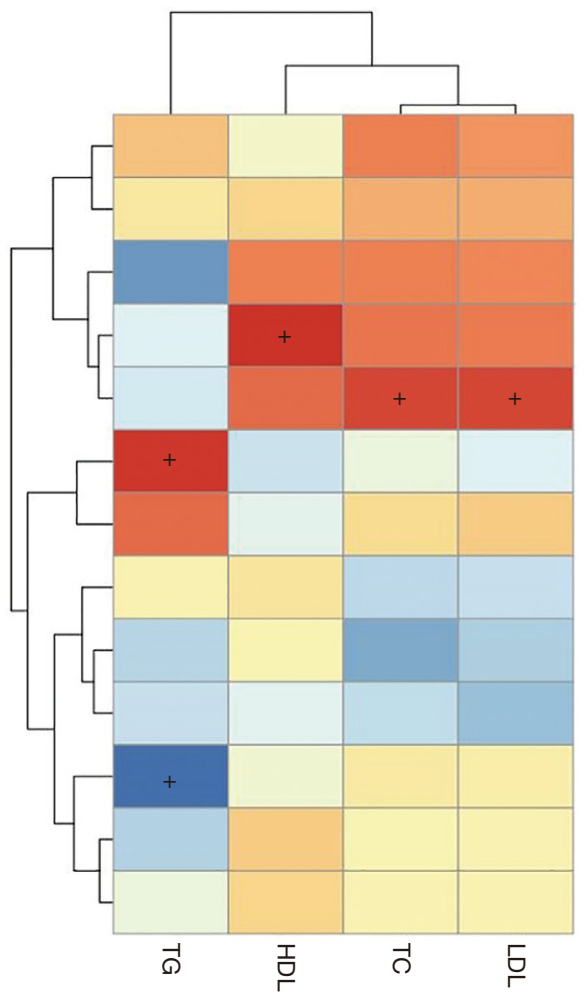

B

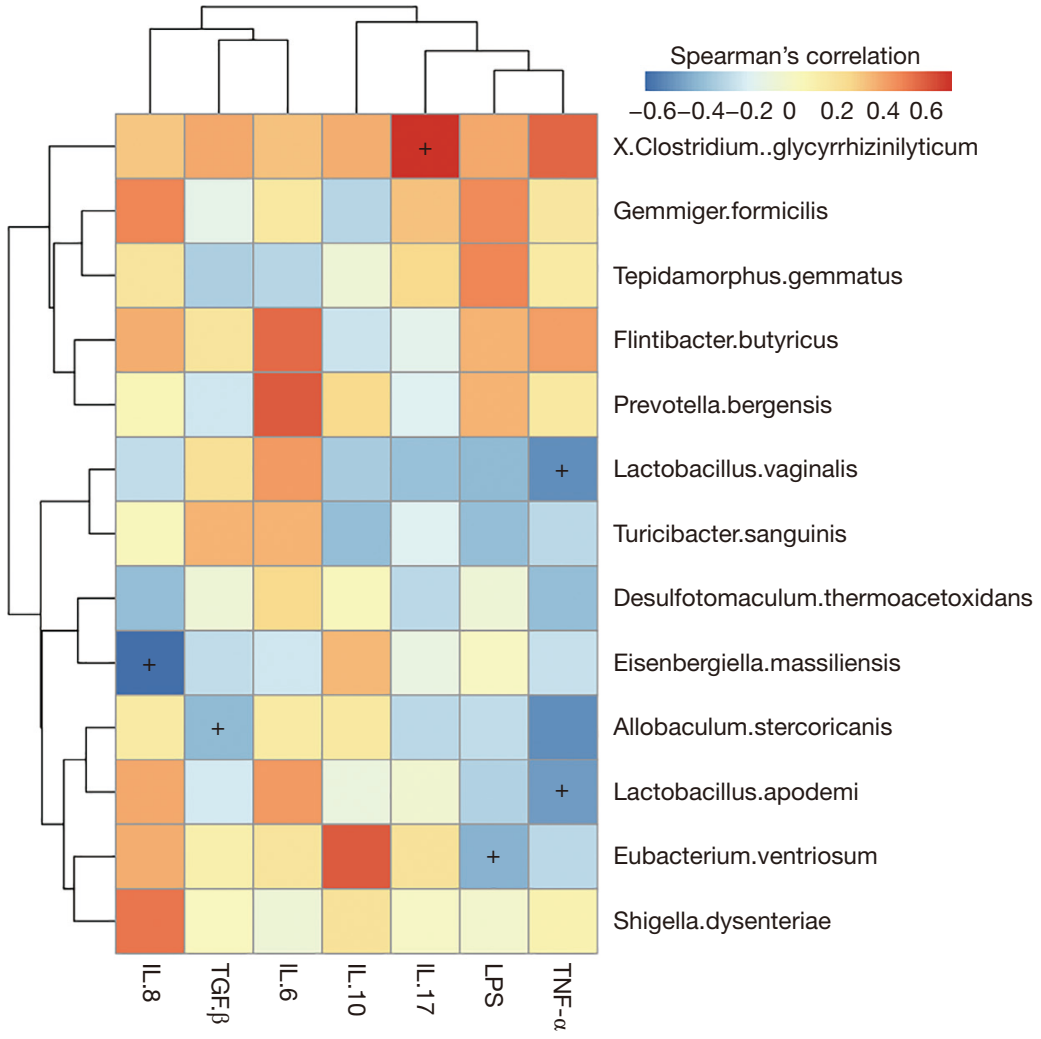

Spearman's correlation

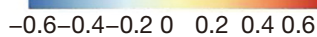

Prevotella.bergensis

Turicibacter.sanguinis

Desulfotomaculum.thermoacetoxidans

Lactobacillus.apodemi

Lactobacillus.vaginalis

X.Clostridium..glycyrrhizinilyticum

Flintibacter.butyricus

Eubacterium.ventriosum

Shigella.dysenteriae

Tepidamorphus.gemmatus

Eisenbergiella.massiliensis

Allobaculum.stercoricanis

Gemmiger.formicilis

Figure 6 Numerical correlation of gut micro flora with blood lipids and cytokines. (A) Numerical correlations between gut microbiota and serum lipids. (B) Numerical correlations between gut microbiota and serum inflammatory factors. Spearman's rank correlation coefficients are indicated by color gradient: red represents a positive correlation; blue represents a negative correlation. $+\mathrm{P}<0.05$. 
gut microbiota modulation. Our results showed that the $\mathrm{L}$. apodemi content in the QHF group was higher than that in the HFD group, and a positive correlation was observed between L. apodemi and HDL level, which is inversely associated with the incidence of heart disease, so it can be inferred that QHF reduces blood lipid levels by regulating the Lactobacillus ratio. Lagkouvardos et al. (56) have established the first gut microbiota database by studying a variety of bacterial communities in the guts of mice. It has been observed that Flintibacter butyricus produces butyrate not only from sugars, but also amino acids. Another study indicated that the HFD-induced gut microbiota imbalance in mice can be corrected with sodium butyrate $(\mathrm{NaB})$, which promotes the abundance of the beneficial bacteria, Blautia and Lactobacillus, that produce butyric acid. Furthermore, compared with an HFD group, $\mathrm{NaB}$ reversed HFDinduced damage to the intestinal mucosa in mice, decreased the levels of gut-derived endotoxins in serum and the liver and increased the expression of ZO-1 in the small intestine. Moreover, liver inflammation and fat accumulation were ameliorated after $\mathrm{NaB}$ administration, while the levels of TG and cholesterol in the liver were reduced and the NAFLD activity score significantly decreased. These findings are consistent with the results from the present study, in which the levels of Flintibacter butyricus and the Blautia luti levels in the QHF group were higher than those in the HFD group, while that in the NC group was similar to that in the QHF groups.

The liver and the intestine originate from the same bacterial layer, maintaining an anatomical and functional connection to a certain extent (19). The gut-liver axis refers to the two-way relationship between the intestine and its microbiota and the liver, which is integrated by environmental characteristics, regional diet, genetics and other related factors, and affects each other (57). Intestinal flora is an important basis for maintaining the homeostasis of the intestinal-liver axis. The intact intestinal mucosa and vascular barrier limit the systemic spread of microorganisms and toxins, while allowing nutrients to enter the circulation and reach the liver $(57,58)$. The imbalance of the intestinal flora will increase the permeability of the intestinal mucosa, so that harmful factors such as endotoxins and inflammatory factors will quickly accumulate in the liver, which will further aggravate the occurrence and development of NAFLD. Similarly, the deterioration of liver disease will also exacerbate the imbalance in the intestinal tract in turn. This study shows that QHF can adjust the distribution and quantity of the intestinal flora of NAFLD rats induced by HFD, increase the abundance of intestinal bacteria and the number of Flintibacter butyricus in the model group, and better maintain the balance of intestinal flora. It can also alleviate serum levels of inflammatory factors such as TNF- $\alpha$, IL-6, IL-8, IL-17 and reduce the accumulation of inflammatory substances in the liver, lower ALT and TG levels, improve liver inflammation and lipid metabolism, and help restore homeostasis of the entero-hepatic axis. In addition, the regulation of enterohepatic circulation is closely related to the metabolism of bile acids. Changes in bile acid products could regulate the body's absorption of food, and affect liver steatosis and glucose absorption. Active transcription factors such as Farnesoid X Receptor (FXR), Liver X Receptor (LXR) and Cholesterol 7 $\alpha$-hydroxylase (CYP7A1) participate in the enterohepatic circulation and change the bile acid metabolism spectrum to regulate glucose and lipid metabolism (59-61). Studies have shown that bile acid supplements can improve fat absorption and increase glucose tolerance (62); administration of intestinal FXR antagonists can improve liver steatosis in mice and help prevent obesity-related metabolic diseases $(60,63)$.

Studies have shown that inflammasomes such as NARP 3 and caspase 1/11 can regulate intestinal flora and lipid distribution, and may be important factors affecting NAFLD lipid deposition and inflammatory lesions (64-66). Caspase-1 and Caspase-11 belong to the classical and nonclassical pathways of pyroptosis, respectively. Both can be activated by NLRP3 to release obesity-related proinflammatory factors IL-18 and IFN- $\beta$, which help to regulate systemic lipid distribution, lipid composition of the liver and the diversity of the intestinal microbial community (66-70). For example, compared with wildtype mice fed high-fat diet, the degree of liver steatosis in $\mathrm{NLRP}^{-/-}$mice fed high-fat diet was reduced. The body weight and subcutaneous fat tissue content of Caspases $1^{-/-}$ and Caspases $11^{-/-}$mice were higher than those of wildtype mice, and the degree of liver steatosis was significantly increased. At the same time, the number of Proteobacteria in the intestine increased, the number of Lactobascillaceae, Verrucomicrobia, and Actinobacteria significantly decreased, while the Firmicutes/Bacteroidetes ratio significantly increased and this could be regarded as a microbial marker signal of obesity and lipid deposition $(11,66,71)$.

Eisenbergiella massiliensis, a new species isolated from human stool samples collected after bariatric surgery (72), displays $97.7 \%$ sequence similarity with E. tayi, which 
is a catalase-positive, rod-shaped, non-proteolytic, nonmotile, anaerobic bacterial strain. This isolate produces butyrate, lactate, acetate and succinate as major metabolic endproducts (73). Our analysis of gut microbiota diversity in rats indicated that species richness in the QHF group was increased compared with that in the HFD group, and the number of firmicutes in the NC group was lower than that in the HFD group at the phylum level. These findings were similar to those in a previous study (74). It is interesting that the abundance of the butyric acid-producing Flintibacter butyricus in the NC group was significantly higher than that in the HFD group. Furthermore, after QHF treatment the abundance of these bacteria in the QHF group increased to that of the NC group. We speculate that the bacterial profiles of HFD-induced rats can be modulated by QHF. Elucidation of these differences in microbiota composition may lead to improved understanding of the pharmacological mechanism underlying the effects of QHF.

\section{Conclusions}

TCM is often used as an alternative medicine and has been shown to have a significant effect on certain conditions, especially chronic diseases (75). Moreover, numerous traditional herbal medicines have long been used in eastern Asia for the treatment of various metabolic disorders (76). However, owing to their complex ingredients and intensive interactions, it is still difficult to explain the specific mechanism of action. Our results from the present study demonstrated that QHF could reduce the inflammation of liver cells, decrease the level of transaminases and improve the fatty deposition in the liver of rats with NAFLD. QHF may modulate the composition of the gut microbiota of NAFLD rats by reducing the abundance of some opportunistic pathogens, such as Clostridium glycyrrbizinilyticum, and increasing butyrate-producing bacteria, such as Flintibacter butyricus and Blautia luti, which may affect the course of the disease to some extent. These results provide a basis for further development of QHF as an alternative approach to treating NAFLD. In the future, we will also expand the number of patients to observe, design more complete clinical studies, combine the different physical types and clinical symptoms of patients, and treat them based on syndrome differentiation, so as to further improve the clinical efficacy and provide more comprehensive diagnosis, treatment plans and clinical evidence for the treatment of NAFLD.

\section{Acknowledgments}

Funding: This work was supported by the funding from the National Natural Science Foundation of China (grant no. 81403357); Shanghai Talents of Chinese Medicine Academic Community Program; the 4th batch of National Chinese Medicine Outstanding Talents Research and Training Project of the State Administration of Traditional Chinese Medicine (China Traditional Chinese Medicine Office [2017] No. 124).

\section{Footnote}

Reporting Checklist: The authors have completed the ARRIVE reporting checklist. Available at http://dx.doi. org/10.21037/apm-21-448

Data Sharing Statement: Available at http://dx.doi. org/10.21037/apm-21-448

Conflicts of Interest: All authors have completed the ICMJE uniform disclosure form (available at http://dx.doi. org/10.21037/apm-21-448). The authors have no conflicts of interest to declare.

Ethical Statement: The authors are accountable for all aspects of the work in ensuring that questions related to the accuracy or integrity of any part of the work are appropriately investigated and resolved. All the procedures for animal use and care in the present study were reviewed and approved by the Animal Ethics Committee of the Medical Center of Fudan University (Shanghai, China; Approval No. 20171717A744). The care and use of animals followed the Guidelines for Animal Experiments of the Medical Center of Fudan University, and all efforts were made to minimize the number of animals and their suffering.

Open Access Statement: This is an Open Access article distributed in accordance with the Creative Commons Attribution-NonCommercial-NoDerivs 4.0 International License (CC BY-NC-ND 4.0), which permits the noncommercial replication and distribution of the article with the strict proviso that no changes or edits are made and the original work is properly cited (including links to both the formal publication through the relevant DOI and the license). See: https://creativecommons.org/licenses/by-nc-nd/4.0/. 


\section{References}

1. Vernon G, Baranova A, Younossi ZM. Systematic review: the epidemiology and natural history of non-alcoholic fatty liver disease and non-alcoholic steatohepatitis in adults. Aliment Pharmacol Ther 2011;34:274-85.

2. Tiniakos DG, Vos MB, Brunt EM. Nonalcoholic fatty liver disease: pathology and pathogenesis. Annu Rev Pathol 2010;5:145-71.

3. Angulo P, Lindor KD. Non-alcoholic fatty liver disease. J Gastroenterol Hepatol 2002;17:S186-90.

4. Zelber-Sagi S, Lotan R, Shlomai A, et al. Predictors for incidence and remission of NAFLD in the general population during a seven-year prospective follow-up. J Hepatol 2012;56:1145-51.

5. Younossi ZM, Koenig AB, Abdelatif D, et al. Global epidemiology of nonalcoholic fatty liver disease-Metaanalytic assessment of prevalence, incidence, and outcomes. Hepatology 2016;64:73-84.

6. Diehl AM, Day C. Cause, Pathogenesis, and Treatment of Nonalcoholic Steatohepatitis. N Engl J Med 2017;377:2063-72.

7. Fan JG, Kim SU, Wong VW. New trends on obesity and NAFLD in Asia. J Hepatol 2017;67:862-73.

8. de Alwis N, Day C. Genetics of Alcoholic Liver Disease and Nonalcoholic Fatty Liver Disease. Semin Liver Dis 2007;27:44-54.

9. Ghaemi A, Taleban FA, Hekmatdoost A, et al. How Much Weight Loss is Effective on Nonalcoholic Fatty Liver Disease? Hepat Mon 2013;13:e15227.

10. Blaut M. Gut microbiota and energy balance: role in obesity. Proc Nutr Soc 2015;74:227-34.

11. Yin J, Li Y, Han H, et al. Administration of exogenous melatonin improves the diurnal rhythms of the gut microbiota in mice fed a high-fat diet. mSystems 2020; 5 : e00002-20.

12. LeBlanc JG, Milani C, de Giori GS, et al. Bacteria as vitamin suppliers to their host: a gut microbiota perspective. Curr Opin Biotechnol 2013;24:160-8.

13. Gallo A, Passaro G, Gasbarrini A, et al. Modulation of microbiota as treatment for intestinal inflammatory disorders: An uptodate. World J Gastroenterol 2016;22:7186-202.

14. Gkolfakis P, Dimitriadis G, Triantafyllou K. Gut microbiota and non-alcoholic fatty liver disease. Hepatobiliary Pancreat Dis Int 2015;14:572-81.

15. Yu ZT. Molecular Mechanism of Intestinal Flore Regulating Lipid Metabolism. Lab Anim Sci 2020,37:74-7.
16. Aron-Wisnewsky J, Vigliotti C, Witjes J, et al. Gut Microbiota and Human NAFLD: Disentangling Microbial Signatures From Metabolic Disorders. Nat Rev Gastroenterol Hepatol 2020;17:279-97.

17. Chen J, Vitetta L. Butyrate in Inflammatory Bowel Disease Therapy. Gastroenterology 2020;158:1511.

18. Wieland A, Frank DN, Harnke B, et al. Systematic review: Microbial dysbiosis and nonalcoholic fatty liver disease. Aliment Pharmacol Ther 2015;42:1051-63.

19. Abu-Shanab A, Quigley EM. The role of the gut microbiota in nonalcoholic fatty liver disease. Nat Rev Gastroenterol Hepatol 2010;7:691-701.

20. Aron-Wisnewsky J, Gaborit B, Dutour A, et al. Gut microbiota and non-alcoholic fatty liver disease: new insights. Clin Microbiol Infect 2013;19:338-48.

21. Li FL, Su YX. Research progress on the relationship between intermittent fasting, intestinal flora and nonalcoholic fatty liver. J Northwest Minzu Univ(Natural Sci) 2020;41:70-3.

22. Nobili V, Putignani L, Mosca A, et al. Bifidobacteria and lactobacilli in the gut microbiome of children with nonalcoholic fatty liver disease: which strains act as health players? Arch Med Sci 2018;14:81-7.

23. Crow JM. Microbiome: That healthy gut feeling. Nature 2011;480:S88-9.

24. Zhang X, Zhao YF, Hu YY, et al. Whole-Body Systems Biology Based on Functional Metagenomics-New Opportunity for the Modernization of Traditional Chinese Medicine. World Science \& Technology 2011;13:202-12.

25. Chen L, Tai WC, Brar MS, et al. Tumor grafting induces changes of gut microbiota in athymic nude mice in the presence and absence of medicinal Gynostemma saponins. PLoS One 2015;10:e0126807.

26. Wang JH, Bose S, Kim HG, et al. Fermented Rhizoma Atractylodis Macrocephalae alleviates high fat dietinduced obesity in association with regulation of intestinal permeability and microbiota in rats. Sci Rep 2015;5:8391.

27. Mei L, Tang Y, Li M, et al. Co-Administration of Cholesterol-Lowering Probiotics and Anthraquinone from Cassia obtusifolia L. Ameliorate Non-Alcoholic Fatty Liver. PLoS One 2015;10:e0138078.

28. Peng JH, Leng J, Tian HJ, et al. Geniposide and Chlorogenic Acid Combination Ameliorates Nonalcoholic Steatohepatitis Involving the Protection on the Gut Barrier Function in Mouse Induced by High-Fat Diet. Front Pharmacol 2018;9:1399.

29. Lu S, Lv SX, Chen Y, et al. Clinical Study on Qinghua Decoction in Treating Non-alcoholic Fatty Liver with 
Phlegm Turbidity Internal Obstruction. Modernization of Trad Chin Med-MST 2019;21:1759-65.

30. Yang DF, Chen CL, Tong HT, et al. Regularities of Medicinal Diet Recipes for Qi-deficiency Constitution in Chinese Medicinal Diet Dictionary. Guid J Tradit Chin Med Pharm 2021;27:171-74.

31. Li Y, Yue WY, Shen TB. Effect of "Yiqi Qinghua Decoction" on intestinal flora in non-alcoholic fatty liver disease rats. Shanghai J Tradit Chin Med 2015;8:79-83.

32. Xu P, Hong F, Wang J, et al. DBZ is a putative PPAR $\gamma$ agonist that prevents high fat diet-induced obesity, insulin resistance and gut dysbiosis. Biochim Biophys Acta Gen Subj 2017;1861:2690-701.

33. Tung YC, Chang WT, Li S, et al. Citrus peel extracts attenuated obesity and modulated gut microbiota in mice with high-fat diet-induced obesity. Food Funct 2018;9:3363-73.

34. Yang DH, Lou ZH, Cheng B, et al. Effects of lotus leaf on inflammatory factors and liver AdipoR2 expressions in rats with NAFLD induced by high fat diet and high glucose. Zhongguo Zhong Yao Za Zhi 2016;41:3406-11.

35. Cope K, Risby T, Diehl AM. Increased gastrointestinal ethanol production in obese mice: implications for fatty liver disease pathogenesis. Gastroenterology 2000;119:1340-7.

36. Nair S, Cope K, Risby TH, et al. Obesity and female gender increase breath ethanol concentration: potential implications for the pathogenesis of nonalcoholic steatohepatitis. Am J Gastroenterol 2001;96:1200-4.

37. Li Z, Yang S, Lin H, et al. Probiotics and antibodies to TNF inhibit inflammatory activity and improve nonalcoholic fatty liver disease. Hepatology 2003;37:343-50.

38. Machado MV, Cortez-Pinto H. Diet, Microbiota, Obesity, and NAFLD: A Dangerous Quartet. Int J Mol Sci 2016;17:481

39. Federico A, Dallio M, Godos J, et al. Targeting gut-liver axis for the treatment of nonalcoholic steatohepatitis: translational and clinical evidence. Transl Res 2016;167:116-24.

40. Kirpich IA, Marsano LS, McClain CJ. Gut-liver axis, nutrition, and non-alcoholic fatty liver disease. Clin Biochem 2015;48:923-30.

41. Vanni E, Bugianesi E. The gut-liver axis in nonalcoholic fatty liver disease: Another pathway to insulin resistance? Hepatology 2009;49:1790-2.

42. Wei X, Zong W, Gao Y, et al. Effects of the Traditional Chinese Medicine Tang Luo Ning on intestinal flora and oxidative stress in diabetic rats. Evid Based Complement Alternat Med 2020;2020:3452625.

43. Singh DP, Singh J, Boparai RK, et al. Isomaltooligosaccharides, a prebiotic, functionally augment green tea effects against high fat diet-induced metabolic alterations via preventing gut dysbacteriosis in mice. Pharmacol Res 2017;123:103-13.

44. Fu JH, Sun HS, Wang Y, et al. The effects of a fat-and sugar-enriched diet and chronic stress on nonalcoholic fatty liver disease in male Wistar rats. Dig Dis Sci 2010;55:2227-36.

45. Zimmermann $M$. Ethical guidelines for investigations of experimental pain in conscious animals. Pain 1983;16:109-10.

46. Boon N, Windt W, Verstraete W, et al. Evaluation of nested PCR-DGGE (denaturing gradient gel electrophoresis) with group-specific $16 \mathrm{~S}$ rRNA primers for the analysis of bacterial communities from different wastewater treatment plants. Fems Microbiol Ecol 2002;39:101-12.

47. Karanth KP, Avivi A, Beharav A, et al. Microsatellite diversity in populations of blind subterranean mole rats (Spalax ehrenbergi superspecies) in Israel: speciation and adaptation. Biol J Linn Soc 2004;83:229-41.

48. Lilleyman J. Practical Statistics for Medical Research. J Clin Pathol 1992;45:368.

49. Porras D, Nistal E, Martínez-Flórez S, et al. Protective effect of quercetin on high-fat diet-induced non-alcoholic fatty liver disease in mice is mediated by modulating intestinal microbiota imbalance and related gut-liver axis activation. Free Radic Biol Med 2017;102:188-202.

50. Everard A, Belzer C, Geurts L, et al. Cross-talk between Akkermansia muciniphila and intestinal epithelium controls diet-induced obesity. Proc Natl Acad Sci U S A 2013;110:9066-71.

51. Erridge C, Attina T, Spickett CM, et al. A high-fat meal induces low-grade endotoxemia: evidence of a novel mechanism of postprandial inflammation. Am J Clin Nutr 2007;86:1286-92.

52. Farhadi A, Gundlapalli S, Shaikh M, et al. Susceptibility to gut leakiness: a possible mechanism for endotoxaemia in non-alcoholic steatohepatitis. Liver Int 2008;28:1026-33.

53. Ruiz AG, Casafont F, Crespo J, et al. Lipopolysaccharidebinding protein plasma levels and liver TNF-alpha gene expression in obese patients: evidence for the potential role of endotoxin in the pathogenesis of non-alcoholic steatohepatitis. Obes Surg 2007;17:1374-80.

54. Sakuma K, Kitahara M, Kibe R, et al. Clostridium 
glycyrrhizinilyticum sp. nov., a glycyrrhizin-hydrolysing bacterium isolated from human faeces. Microbiol Immunol 2006;50:481-5.

55. Lye HS, Kato T, Low WY, et al. Lactobacillus fermentum FTDC 8312 combats hypercholesterolemia via alteration of gut microbiota. J Biotechnol 2017;262:75-83.

56. Lagkouvardos I, Pukall R, Abt B, et al. The Mouse Intestinal Bacterial Collection (miBC) provides hostspecific insight into cultured diversity and functional potential of the gut microbiota. Nat Microbiol 2016;1:16131.

57. Meng QH, OuYang XH, Su LX. Research progress in the treatment and prevention of entero-hepatic axis and hepatocellular carcinoma. Chin Preventive Med 2020;21:1149-52.

58. Li L, Wang YL, Qin HY, et al. Research progress on berberine in treatment of nonalcoholic fatty liver disease by regulating gut-liver axis. Chin Trad and Herbal Drugs 2021;52:1501-9.

59. Zheng D, Liwinski T, Elinav E. Interaction between microbiota and immunity in health and disease. Cell Res 2020;30:492-506.

60. Sun L, Pang Y, Wang X, et al. Ablation of gut microbiota alleviates obesity-induced hepatic steatosis and glucose intolerance by modulating bile acid metabolism in hamsters. Acta Pharm Sin B 2019;9:702-10.

61. Duparc T, Plovier H, Marrachelli VG, et al. Hepatocyte MyD88 affects bile acids, gut microbiota and metabolome contributing to regulate glucose and lipid metabolism. Gut 2017;66:620-32.

62. Wang DQ, Tazuma S, Cohen DE, et al. Feeding natural hydrophilic bile acids inhibits intestinal cholesterol absorption: studies in the gallstone-susceptible mouse. Am J Physiol Gastrointest Liver Physiol 2003;285:G494-502.

63. Gonzalez FJ, Jiang C, Patterson AD. An intestinal microbiota-farnesoid $\mathrm{X}$ receptor axis modulates metabolic disease. Gastroenterology 2016;151:845-59.

64. Wree A, Eguchi A, McGeough MD, et al. NLRP3 inflammasome activation results in hepatocyte pyroptosis, liver inflammation, and fibrosis in mice. Hepatology 2014;59:898-910.

65. Henao-Mejia J, Elinav E, Jin C, et al. Inflammasomemediated dysbiosis regulates progression of NAFLD and obesity. Nature 2012;482:179-85.

66. de Sant'Ana LP, Ribeiro DJS, Martins AMA, et al. Absence of the Caspases 1/11 Modulates Liver Global Lipid Profile and Gut Microbiota in High-Fat-Diet-Induced Obese Mice. Front Immunol 2020;10:2926.

67. Noureddin M, Sanyal A J. Pathogenesis of NASH: The Impact of Multiple Pathways. Curr Hepatol Rep 2018;17:350-60.

68. Lin Y. Role of NLRP3 Mediated Mitochondrial Damage in Non-alcoholic Steatohepatitis and Intervention of SS31. Med Innovation Chin 2020;17:23-8.

69. Zhu DQ, Li PZ. Role of Kupffer cells in bacterial infectious diseases. World Chin J Dig 2015;23:1776-83.

70. Ballak DB, Stienstra R, Tack CJ, et al. IL-1 family members in the pathogenesis and treatment of metabolic disease: focus on adipose tissue inflammation and insulin resistance. Cytokine 2015;75:280-90.

71. Dixon LJ, Flask CA, Papouchado BG, et al. Caspase-1 as a central regulator of high fat diet-induced non-alcoholic steatohepatitis. PLoS ONE 2013;8:e56100.

72. Togo AH, Khelaifia S, Bittar F, et al. 'Eisenbergiella massiliensis', a new species isolated from human stool collected after bariatric surgery. New Microbes New Infect 2016;13:15-6.

73. Bernard K, Burdz T, Wiebe D, et al. Characterization of isolates of Eisenbergiella tayi, a strictly anaerobic Gramstain variable bacillus recovered from human clinical materials in Canada. Anaerobe 2017;44:128-32.

74. Ushijima Y, Tajima T, Yoshimitsu K, et al. Radiological catheter placement for transcatheter arterial steroid injection therapy to treat severe acute hepatic failure: technical feasibility and efficacy. Acta Radiol 2012;53:140-6.

75. Normile D. Asian medicine. The new face of traditional Chinese medicine. Science 2003;299:188-90.

76. Yin J, Zhang H, Ye J. Traditional chinese medicine in treatment of metabolic syndrome. Endocr Metab Immune Disord Drug Targets 2008;8:99-111.

(English Language Editor: K. Brown)

Cite this article as: Wang Y, Lv S, Shen T, Ye M, Wang D, Zhou P, Li Y, Zhang W. Qinghua Fang inhibits high-fat diet-induced non-alcoholic fatty liver disease by modulating gut microbiota. Ann Palliat Med 2021;10(3):3219-3234. doi: 10.21037/apm-21-448 